\title{
Endoscopic transaxillary dual-plane breast augmentation: a four-year retrospective clinical study
}

\author{
Jeong-Ho Seo, MD, PhD \\ Department of Plastic Surgery, Yoonho Hospital, Seoul, Rep. of Korea
}

Background: Endoscopic transaxillary dual plane breast augmentation is currently the treatment of choice of breast augmentation. Although the dual-plane pocket technique yields excellent outcomes, there are few reports that describe a dual-plane transaxillary approach technique using an endoscope.

Objective: The purpose of this study was to evaluate the results and complications of patients who had undergone endoscopic transaxillary dual-plane breast augmentation.

Methods: The patients who had undergone transaxillary dual-plane breast augmentation using endoscope from January 2014 to December 2017 were reviewed.

Results: A total of 58 patients underwent endoscopic dual-plane breast augmentation through the axillary incision approach. Of these, only one patient (1.7\%) showed bilateral superior dislocation as a complication, which was treated by endoscopic transaxillary capsulotomy. No serious complications were reported during the course of this study. All patients were discharged on day one postoperatively and recovered quickly.

Conclusion: Endoscopic dual-plane pocket breast augmentation through the axillary approach is a safe operation, has a low complication rate, and yields satisfactory results in the patients.

Keywords: axillary approach; breast augmentation; dual-plane; endoscopes

\section{Introduction}

Breast augmentation is one of the most common aesthetic surgery procedures worldwide [1]. Conventional dual-plane pocket breast augmentation generally involves incisions in the inframammary or periareolar creases. However, particularly in Korean women, these types of incisions leave distinct and hypertrophic scars on the breast skin. When a patient prefers not to have a scar on the breast, it is necessary to perform transaxillary dual-plane breast augmentation using the endoscopic technique.

Dual-plane pocket breast augmentation incorporates the ad- vantages and eliminates the disadvantages of subglandular and submuscular plane operations [2]. In this study, we analyzed the effectiveness and safety of endoscopic dual-plane breast augmentation surgery through the axillary approach.

\section{Materials and methods}

From January 2014 to December 2017, a total of 58 patients underwent endoscopic dual-plane pocket breast augmentation through the axillary approach. The procedure was performed under general anesthesia on a one-day hospitalization basis.

Soft cohesive silicone gel-textured or micro-textured, round

Received October 15, 2018; Revised November 7, 2018; Accepted November 22, 2018

Corresponding author: Jeong-Ho Seo

E-mail: drseokr@naver.com, ORCID: https://orcid.org/0000-0003-1466-2139

This is an Open Access article distributed under the terms of the Creative Commons Attribution Non-Commercial License (http://creativecommons.org/licenses/by-nc/4.0), which permits unrestricted non-commercial use, distribution, and reproduction in any medium, provided the original work is properly cited.

Copyright (c) 2018 Korean Society of Korean Cosmetic Surgery and Medicine (KSKCS \& KCCS). 
implants ranging in size from $180 \mathrm{ml}$ to $280 \mathrm{ml}$ were used (Sebbin, Boissy-l'Aillerie, France; Allergan, Madison, NJ, USA).

\section{Surgical technique}

Preoperative skin markings are made with the patient in the standing and supine positions. The nipple areolar complex is the anatomic landmark for the center of the breast. The patient is placed supine with both arms abducted ninety degrees. A mixture including $50 \mathrm{ml}$ of normal saline, $5 \mathrm{ml}$ of $2 \%$ lidocaine, and $0.25 \mathrm{ml}$ of epinephrine is injected into the subcutaneous tissue and at the junction of the lateral border of the pectoralis major muscle fascia on each side to decrease subcutaneous bleeding.

A 4- or 5-cm horizontal incision in the axilla is made following Langer's lines $1 \mathrm{~cm}$ posterior to anterior axillary line. The lateral pectoral fascia is incised using electrocautery to identify the lateral border of the pectoralis major muscle. A pocket between the pectoralis major and pectoralis minor muscles is dissected and created using an endoscope to visualize the operative field and endoscopic ligasure to dissect the operative field. A $10 \mathrm{~mm}$, $0^{\circ}$ or $30^{\circ}$ endoscope (Richard Wolf $\mathrm{GmbH}$, Knittlingen, Germany) and Ligasure (Valley Lab LS10, Covidienm, Mamsfield, MA, USA) are used to dissect and coagulate the pocket site between the pectoralis major and minor muscles.

With an endoscope and Ligasure, meticulous dissection and coagulation are performed with repeated assessments in rela- tion to the external skin marking, while the pectoralis major muscle is cut free from the pectoralis minor muscle. During muscle detachment, caution must be observed not to extend the medial origins of the pectoralis major muscle. The dissection plane should never extend beyond the anterior axillary line; this precaution will prevent lateral displacement of the implant.

Precise hemostasis is performed using an endoscope and by Ligasure bipolar cauterization. The optimal direction to enter the submuscular plane is a point halfway between the nipple and the clavicle in a direction inferior to the incision. By releasing the pectoralis major muscle along the inframammary fold in the lower pole, the medial origins superior to the junction of the inframammary fold with the sternum is not divided to his prevent complications such as symmastia, traction rippling, and banding deformity.

A round textured silicone cohesive gel implant is inserted through the axillary incision. An anatomic-type implant is not used because of the difficulty inprecise implant placement. The incision is closed using 3-0 vicryl (Ethicon, Somerville, NJ, USA) for subcutaneous tissue and 5-0 nylon for axillary skin. No indwelling drains are placed. An elastic compressive brassiere is placed following elastic plaster application on the upper pole of the breast.

On postoperative day 1 , all patients are discharged and are evaluated by preoperative and postoperative photographic
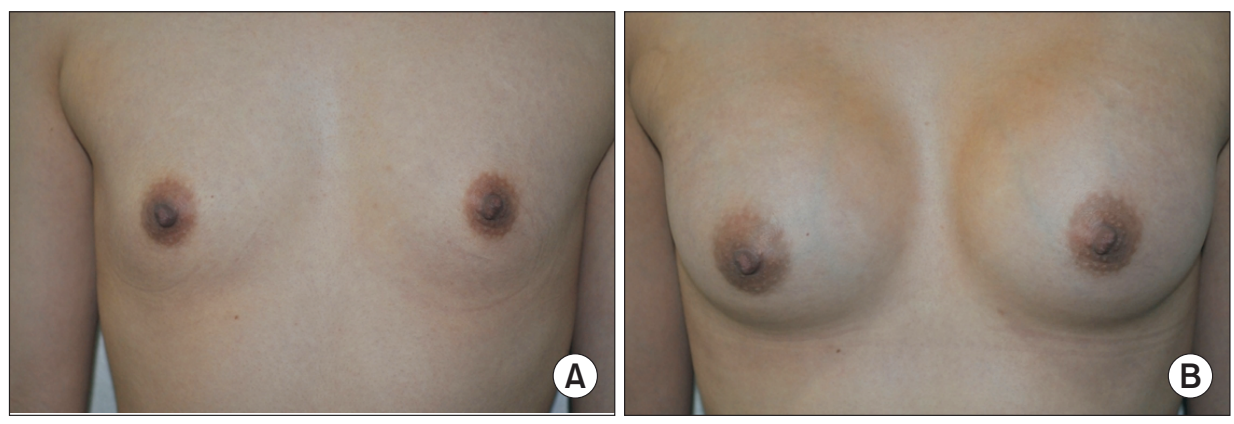

Fig. 1. (A) Preoperative view of a 28-yearold patient. (B) Postoperative view 1 month following the operation.
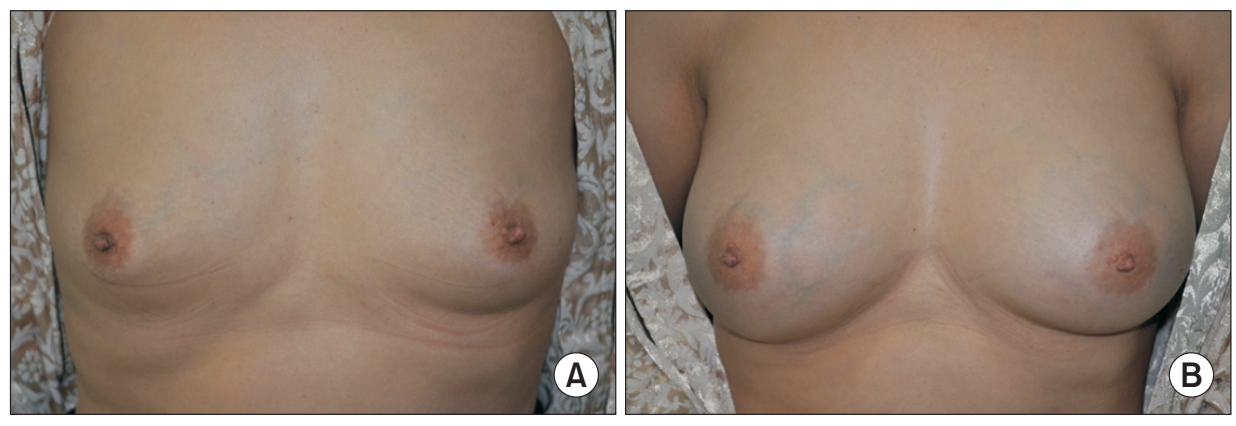

Fig. 2. (A) Preoperative view of a 38-yearold patient. (B) Postoperative 6 months following the operation. 

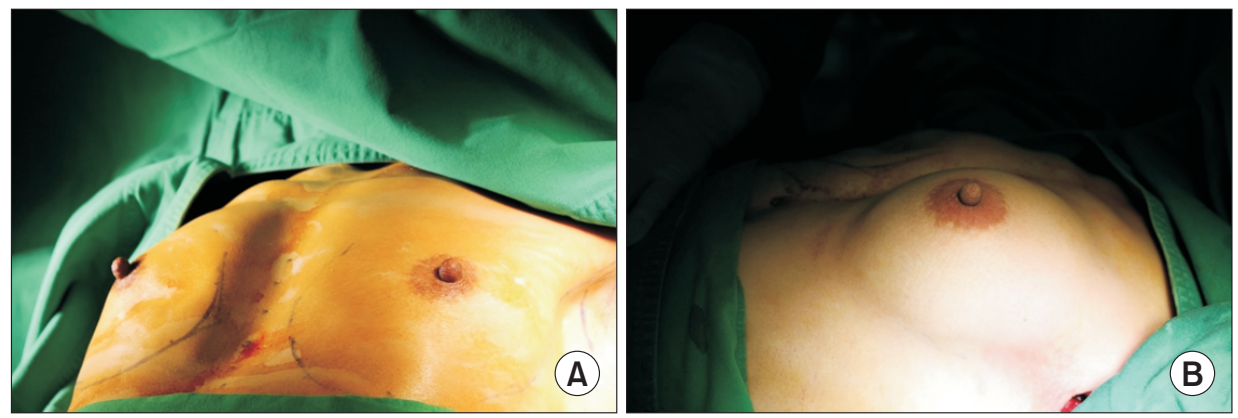

Fig. 3. (A) Preoperative view of a 45-yearold patient. (B) Postoperative view just after the operation.

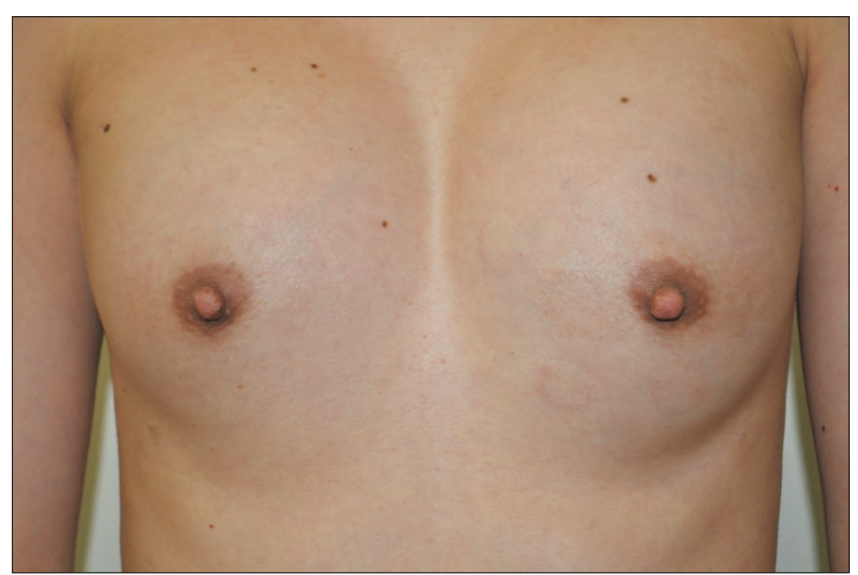

Fig. 4. The patient with the complication of inferior pocket closure following primary breast augmentation 6 months later.

analysis (Fig. 1-3). All patients are requested to return for follow up at one week, one month, two months, and six months thereafter.

\section{Results}

Fifty-eight patients underwent endoscopic dual-plane breast augmentation through the axillary approach and were discharged from the hospital on day one postoperatively. The mean age of the patients was 38.3 years (range, 22-56) and the median duration of the operation was 110.4 minutes (range, 90-130).

All patients were followed postoperatively and reported satisfaction with the outcome of the surgery. Only one patient experienced the complication of superior implant malposition due to inferior pocket closure. This superior dislocation was managed by an endoscopic capsulotomy procedure 6 months after the first operation. Of the 58 patients, the 57 patients $(98.3 \%)$ without complication were satisfied with the outcome.

The patient who developed inferior pocket closure as a complication underwent endoscopic transaxillary inferior capsu-

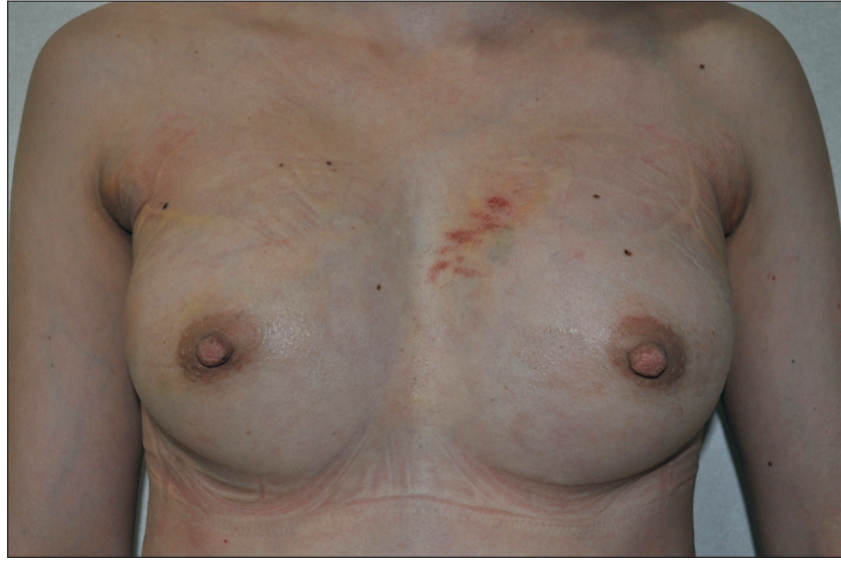

Fig. 5. Post reoperation day 1 figure using endoscopic capsulotomy.

lotomy on both sides. This patient had soft and pliable capsules around all breast implants. Both implants were removed, and inferior capsulotomies were performed simultaneously (Fig. 4, 5).

\section{Discussion}

Endoscopic transaxillary breast augmentation operations have been reported in many articles [3-6]. This procedure initially involved a subglandular plane pocket operation. Nowadays, the endoscopic dual-plane transaxillary breast augmentation procedure remains the standard approach for the vast majority of surgeons [7].

Dual-plane pocket breast augmentation is the best surgical procedure. A dual-plane pocket consists of a subglandular pocket inferiorly and a submuscular pocket superiorly. This procedure has the advantages of subglandular and submuscular plane procedures and eliminates the disadvantages of those procedures. The dual-plane technique enhances the projection of the lower pole and facilitates a more natural appearance. Thus, in this study, we could use only a round-type implant instead of an anatomic type. Another advantage is that optimal 
soft tissue coverage using a dual-plane pocket markedly reduces overall reoperation rates and reoperations for visible rippling [2].

The breast augmentation procedure through the axillary approach was first reported by Hoehler [8]. This approach has been popular because of its aesthetic outcomes. The scars in this procedure are located outside the breast skin. Most surgeons prefer the other incision approaches to the transaxillary approach particularly because this approach technique is more difficult and time consuming than in the other techniques. Many surgeons opt for the periareolar or inframammary fold crease incision approach instead. However, most Asian women, especially Korean women, who want to undergo breast mammaplasty prefer the transaxillary approach procedure to the other incision procedures because the scars are located outside the breast skin [7].

On the basis of this study, Major reasons for using the endoscopic technique is to perform the dual-plane procedure through the axillary approach, and another reason for using an endoscope is to accomplish the prospective hemostasis that prevents blood staining of tissues. Prospective hemostasis is performed meticulously using the endoscopic technique. Micro-hematoma after blind dissection between the pectoralis major and pectoralis minor muscle is one of the main causes of capsular contracture. Therefore, meticulous dissection using the endoscopic technique does not result in a micro-hematoma in the operative field. This is the reason why endoscopic transaxillary breast mammaplasty has a lower rate of capsular contracture complications than do the other breast augmentation operations.

In our study, we experienced one case of inferior pocket closure. After breast augmentation, some degree of dead space exists around the implant. Small amounts of serosanguinous fluid accumulate immediately postoperatively; this wound healing mechanism attempts to close dead space, so many bodies resorb these fluids spontaneously. However, if greater accumulations of fluid in the inframammary fold of dead space occurs, the inflammatory response increases and the fluid accumulated may undergo fibrous replacement that obliterates the inferior dead space. This closes the inferior pocket which is then filled with fibrous tissue or a thick capsule [9].

Inferior pocket closure can occur as an isolated process that does not progress to clinically apparent capsular contracture, but nevertheless, it causes enough superior implant dislocation to create visually apparent differences in upper pole fill [9].
In conclusion, Dual-plane pocket breast augmentation surgery through axillary approach can be accomplished using an endoscopic technique. This procedure is a safe operation, has a low complication rate, and yields satisfactory results in patients.

Therefore, we should recommend endoscopic transaxillary dual-plane breast augmentation. However, no prospective studies and long-term follow up studies have reported this technique using an endoscope, so further evaluation on this topic is needed.

\section{Conflicts of interest}

The author has nothing to disclose.

\section{References}

1. Lang Stümpfle R, Figueras Pereira-Lima L, Alves Valiati A, da Silva Mazzini G. Tansaxillary muscle-splitting breast augmentation: experience with 160 cases. Aesthetic Plast Surg 2012;36:343-8.

2. Tebbetts JB. Dual plane breast augmentation: optimizing implant-soft-tissue relationships in a wide range of breast types. Plast Reconstr Surg 2001;107:1255-72.

3. Ho LC. Endoscopic assisted transaxillary augmentation mammaplasty. Br J Plast Surg 1993;46:332-6.

4. Serra-Renom J, Garrido MF, Yoon T. Augmentation mammaplasty with anatomic soft, cohesive silicone implant using the transaxillary approach at a subfascial level with endoscopic assistance. Plast Reconstr Surg 2005;116:640-5.

5. Momeni A, Padron NT, Bannasch H, Borges J, Björn Stark G. Endoscopic transaxillary subpectoral augmentation mammoplasty: a safe and predictable procedure. J Plast Reconstr Aesthet Surg 2006;59:1076-81.

6. Villafane O, Garcia-Tutor E, Taggart I. Endoscopic transaxillary subglandular breast augmentation using silicone gel textured implants. Aesthetic Plast Surg 2000;24:212-5.

7. Lee SH, Yoon WJ. Axillary endoscopic subglandular tunneling approach for types 2 and 3 dual plane breast augmentation. Aesthetic Plast Surg 2014;38:521-7.

8. Hoehler H. Breast augmentation: the axillary approach. Br J Plast Surg 1973;26:373-6.

9. Tebbetts JB. Augmentation mammoplasty, redefining the patient and surgeon experience. Edinburgh: Mosby Elsevier; 2010. p. 429-31. 